\title{
Harmonization and Standardization of Panel-Based Tumor Mutational Burden (TMB) Measurement: Real-World Results and Recommendations of the QuIP Study
}

\author{
Stenzinger, Albrecht ; Endris, Volker ; Budczies, Jan ; et al ; Moch, Holger
}

\begin{abstract}
INTRODUCTION Tumor mutational burden (TMB) is a quantitative assessment of the number of somatic mutations within a tumor genome. Immunotherapy benefit has been associated with TMB assessed by whole exome sequencing (wesTMB) and by gene panel sequencing (psTMB). The initiatives of Quality in Pathology (QuIP) and Friends of Cancer Research (FoCR) have jointly addressed the need for harmonization between TMB testing options in tissues. This QuIP study identifies critical sources of variation in psTMB assessment. METHODS Twenty samples from three tumor types (LUAD, HNSC, COAD) with available WES data were analyzed for psTMB, using six panels across 15 testing centers. Inter-laboratory and inter-platform variation including agreement on variant calling and TMB classification were investigated. Bridging factors to transform psTMB to wesTMB values were empirically derived. The impact of germline filtering was evaluated. RESULTS Sixteen samples demonstrated low interlaboratory and interpanel psTMB variation with $87.7 \%$ of pairwise comparisons showing a Spearman's >0.6. A wesTMB cutpoint of 199 missense mutations projected to psTMB cutpoints between 7.8 and 12.6 muts/Mbp; the corresponding psTMB and wesTMB classifications agreed in $74.9 \%$ of cases. For three-tier classification with cutpoints of 100 and 300 mutations, agreement was observed in $76.7 \%$, weak misclassification in $21.8 \%$, and strong misclassification in $1.5 \%$ of cases. Confounders of psTMB estimation included fixation artifacts, DNA input, sequencing depth, genome coverage, and variant allele frequency cutpoints. CONCLUSIONS This study provides real-world evidence that all evaluated panels can be used to estimate TMB in a routine diagnostic setting and identifies important parameters for reliable tissue TMB assessment that require careful control. As complex/composite biomarkers beyond TMB are likely playing an increasing role in therapy prediction, the efforts by QuIP and FoCR also delineate a general framework and blueprint for the evaluation of such assays.
\end{abstract}

DOI: https://doi.org/10.1016/j.jtho.2020.01.023

Posted at the Zurich Open Repository and Archive, University of Zurich

ZORA URL: https://doi.org/10.5167/uzh-186492

Journal Article

Accepted Version

Originally published at:

Stenzinger, Albrecht; Endris, Volker; Budczies, Jan; et al; Moch, Holger (2020). Harmonization and Standardization of Panel-Based Tumor Mutational Burden (TMB) Measurement: Real-World Results and Recommendations of the QuIP Study. Journal of Thoracic Oncology, 15(7):1177-1189.

DOI: https://doi.org/10.1016/j.jtho.2020.01.023 


\section{Journal Pre-proof}

Harmonization and Standardization of Panel-Based Tumor Mutational Burden (TMB) Measurement: Real-World Results and Recommendations of the QuIP Study

Albrecht Stenzinger, Volker Endris, Jan Budczies, Sabine Merkelbach-Bruse, Daniel Kazdal, Wolfgang Dietmaier, Nicole Pfarr, Udo Siebolts, Michael Hummel, Sylvia Herold, Johanna Andreas, Martin Zoche, Lars Tögel, Eugen Rempel, Jörg Maas, Diana Merino, Mark Stewart, Karim Zaoui, Matthias Schlesner, Hanno Glimm, Stefan Fröhling, Jeff Allen, David Horst, Gustavo Baretton, Claudia Wickenhauser, Markus Tiemann, Matthias Evert, Holger Moch, Thomas Kirchner, Reinhard Büttner, Peter Schirmacher, Andreas Jung, Florian Haller, Wilko Weichert, Manfred Dietel

PII: $\quad$ S1556-0864(20)30135-0

DOI: $\quad$ https://doi.org/10.1016/j.jtho.2020.01.023

Reference: $\quad$ JTHO 1726

To appear in: Journal of Thoracic Oncology

Received Date: 15 November 2019

Revised Date: 10 January 2020

Accepted Date: 20 January 2020

Please cite this article as: Stenzinger A, Endris V, Budczies J, Merkelbach-Bruse S, Kazdal D, Dietmaier W, Pfarr N, Siebolts U, Hummel M, Herold S, Andreas J, Zoche M, Tögel L, Rempel E, Maas J, Merino D, Stewart M, Zaoui K, Schlesner M, Glimm H, Fröhling S, Allen J, Horst D, Baretton G, Wickenhauser C, Tiemann M, Evert M, Moch H, Kirchner T, Büttner R, Schirmacher P, Jung A, Haller F, Weichert W, Dietel M, Harmonization and Standardization of Panel-Based Tumor Mutational Burden (TMB) Measurement: Real-World Results and Recommendations of the QuIP Study, Journal of Thoracic Oncology (2020), doi: https://doi.org/10.1016/j.jtho.2020.01.023.

This is a PDF file of an article that has undergone enhancements after acceptance, such as the addition of a cover page and metadata, and formatting for readability, but it is not yet the definitive version of record. This version will undergo additional copyediting, typesetting and review before it is published in its final form, but we are providing this version to give early visibility of the article. Please note that, during the production process, errors may be discovered which could affect the content, and all legal disclaimers that apply to the journal pertain. 
(C) 2020 International Association for the Study of Lung Cancer. Published by Elsevier Inc. All rights reserved. 


\section{Harmonization and Standardization of Panel-Based Tumor Mutational \\ Burden (TMB) Measurement: Real-World Results and Recommendations of the QulP Study}

Albrecht Stenzinger ${ }^{\mathrm{a}, \mathrm{b}, \#, *}$, Volker Endris ${ }^{\mathrm{a}, *}$, Jan Budczies ${ }^{\mathrm{a}, \star}$, Sabine Merkelbach-Bruse ${ }^{\mathrm{c}}$, Daniel Kazdal ${ }^{\mathrm{a}, \mathrm{b}}$, Wolfgang Dietmaier ${ }^{\mathrm{d}}$, Nicole Pfarr ${ }^{\mathrm{e}}$, Udo Siebolts ${ }^{f}$, Michael Hummel ${ }^{\mathrm{g}}$, Sylvia Herold ${ }^{h}$, Johanna Andreas', Martin Zochej, Lars Tögel ${ }^{k}$, Eugen Rempel ${ }^{\mathrm{a}}$, Jörg Maas', Diana Merino $^{m}$, Mark Stewart ${ }^{m}$, Karim Zaoui $^{n}$, Matthias Schlesner ${ }^{\circ}$, Hanno Glimm ${ }^{p}$, Stefan Fröhling ${ }^{q}$, Jeff Allen ${ }^{m}$, David Horst ${ }^{q}$, Gustavo Baretton ${ }^{h}$, Claudia Wickenhauser ${ }^{f}$, Markus Tiemanni, Matthias Evert ${ }^{d}$, Holger Moch ${ }^{j}$, Thomas Kirchner ${ }^{r}$, Reinhard Büttner ${ }^{c}$, Peter Schirmacher ${ }^{\mathrm{a}}$, Andreas Jung ${ }^{r}$, Florian Haller ${ }^{\mathrm{k}}$, Wilko Weichert ${ }^{\mathrm{e}}$, Manfred Dietel'

anstitute of Pathology, University Hospital Heidelberg, Heidelberg, Germany

${ }^{b}$ Translational Lung Research Center (TLRC) Heidelberg, German Center for Lung Research (DZL), Heidelberg, Germany

'Institute of Pathology, University Hospital Cologne, Cologne, Germany

'Institute of Pathology, University Regensburg, Regensburg, Germany

e Institute of Pathology, Technical University Munich (TUM), Munich, Germany

Institute of Pathology, University Hospital Halle, Halle, Germany

Institute of Pathology, Charité University Hospital, Berlin, Germany

hInstitute of Pathology, University Hospital Dresden, Dresden, Germany

'Institute of Hematopathology, Hamburg, Germany

'Institute of Pathology, University Hospital Zurich, Zurich, Switzerland

${ }^{k}$ Institute of Pathology, University Hospital Erlangen, Erlangen, Germany

'Quality in Pathology (QuIP), Berlin, Germany

${ }^{\mathrm{m}}$ Friends of Cancer Research (FoCR), Washington, DC

${ }^{n}$ Department of Otorhinolaryngology, University Hospital Heidelberg, Heidelberg, Germany

${ }^{\circ}$ Bioinformatics and Omics Data Analytics, German Cancer Research Center (DKFZ), Heidelberg,

Germany

${ }^{\mathrm{P}}$ Department of Translational Medical Oncology, National Center for Tumor Diseases (NCT Dresden) and University Hospital Carl Gustav Carus, Dresden, and Translational Functional Cancer Genomics, German Cancer Research Center (DKFZ), Heidelberg, Germany and German Cancer Consortium (DKTK), Dresden, Germany

'Division of Translational Medical Oncology, National Center for Tumor Diseases (NCT) Heidelberg and German Cancer Research Center (DKFZ), Heidelberg, Germany

'Institute of Pathology, Ludwig-Maximilians University (LMU), Munich, Germany

${ }^{*}$ Equal contribution

\#Correspondence to

Albrecht Stenzinger, Institute of Pathology, University Hospital Heidelberg, Im Neuenheimer Feld 224, 69120 Heidelberg, Germany 
Phone: +4962215634380

Journal Pre-proof

Email: albrecht.stenzinger@med.uni-heidelberg.de 


\begin{tabular}{|c|c|c|c|}
\hline Author full name & Email address & $\begin{array}{l}\text { Academic } \\
\text { degree }\end{array}$ & $\begin{array}{l}\text { Academic } \\
\text { ranking }\end{array}$ \\
\hline Albrecht Stenzinger & Albrecht.Stenzinger@med.uni-heidelberg.de & MD & Prof \\
\hline Volker Endris & volker.endris@med.uni-heidelberg.de & $\mathrm{PhD}$ & $\mathrm{Dr}$ \\
\hline Jan Budczies & ian.budczies@med.uni-heidelberg.de & $\mathrm{PhD}$ & PD Dr \\
\hline Sabine Merkelbach-Bruse & Sabine.Merkelbach-Bruse@uk-koeln.de & $\mathrm{PhD}$ & Prof \\
\hline Wolfgang Dietmaier & wolfgang.dietmaier@klinik.uni-regensburg.de & $\mathrm{PhD}$ & Prof \\
\hline Nicole Pfarr & Nicole.pfarr@tum.de & $\mathrm{PhD}$ & $\mathrm{Dr}$ \\
\hline Udo Siebolts & udo.siebolts@uk-halle.de & MD, PhD & PD Dr Dr \\
\hline Michael Hummel & Michael.Hummel@charite.de & $\mathrm{PhD}$ & Prof \\
\hline Johanna Andreas & andreas@hp-hamburg.de & B.Sc. & \\
\hline Sylvia Herold & Sylvia.Herold@uniklinikum-dresden.de & $\mathrm{PhD}$ & $\mathrm{Dr}$ \\
\hline Martin Zoche & martin.zoche@usz.ch & $\mathrm{PhD}$ & $\mathrm{Dr}$ \\
\hline Daniel Kazdal & daniel.kazdal@med.uni-heidelberg.de & $\mathrm{PhD}$ & $\mathrm{Dr}$ \\
\hline Jörg Maas & maas@quip.eu & Dipl.-Pol. & \\
\hline Diana M. Merino & dmerino@focr.org & $\mathrm{PhD}$ & $\mathrm{Dr}$ \\
\hline Mark Stewart & mstewart@focr.org & $\mathrm{PhD}$ & $\mathrm{Dr}$ \\
\hline Jeff Allen & jallen@focr.org & $\mathrm{PhD}$ & $\mathrm{Dr}$ \\
\hline Karim Zaoui & karim.zaoui@med.uni-heidelberg.de & MD & $\mathrm{Dr}$ \\
\hline Matthias Schlesner & m.schlesner@dkfz-Heidelberg.de & $\mathrm{PhD}$ & $\mathrm{Dr}$ \\
\hline Hanno Glimm & hanno.glimm@nct-dresden.de & MD & Prof \\
\hline David Horst & David.horst@charite.de & MD & Prof \\
\hline Markus Tiemann & mtiemann@hp-hamburg.de & MD & $\mathrm{Dr}$ \\
\hline Matthias Evert & matthias.evert@klinik.uni-regensburg.de & MD & Prof \\
\hline Holger Moch & holger.moch@usz.ch & MD & Prof \\
\hline Thomas Kirchner & Thomas.Kirchner@med.uni-muenchen.de & MD & Prof \\
\hline Reinhard Büttner & reinhard.buettner@uk-koeln.de & MD & Prof \\
\hline Peter Schirmacher & Peter.Schirmacher@med.uni-heidelberg.de & MD & Prof \\
\hline Eugen Rempel & Eugen.rempel@med.uni-heidelberg.de & $\mathrm{PhD}$ & $\mathrm{Dr}$ \\
\hline Andreas Jung & Andreas.Jung@med.uni-muenchen.de & MD & Prof \\
\hline Florian Haller & florian.haller@uk-erlangen.de & MD & Prof \\
\hline Lars Tögel & Lars.Toegel@uk-erlangen.de & $\mathrm{PhD}$ & $\mathrm{Dr}$ \\
\hline Wilko Weichert & wilko.weichert@tum.de & MD & Prof \\
\hline Manfred Dietel & manfred.dietel@charite.de & MD & Prof \\
\hline Stefan Fröhling & stefan.froehling@nct-heidelberg.de & MD & Prof \\
\hline Gustavo Baretton & gustavo.baretton@uniklinikum-dresden.de & MD & Prof \\
\hline Claudia Wickenhauser & claudia.wickenhauser@uk-halle.de & MD & Prof \\
\hline
\end{tabular}




\section{Abstract (286 words)}

\section{Introduction}

Tumor mutational burden (TMB) is a quantitative assessment of the number of somatic mutations within a tumor genome. Immunotherapy benefit has been associated with TMB assessed by whole exome sequencing (wesTMB) and by gene panel sequencing (psTMB). The initiatives of Quality in Pathology (QuIP) and Friends of Cancer Research (FoCR) have jointly addressed the need for harmonization between TMB testing options in tissues. This QuIP study identifies critical sources of variation in psTMB assessment.

\section{Methods}

Twenty samples from three tumor types (LUAD, HNSC, COAD) with available WES data were analyzed for psTMB, using six panels across 15 testing centers. Inter-laboratory and inter-platform variation including agreement on variant calling and TMB classification were investigated. Bridging factors to transform psTMB to wesTMB values were empirically derived. The impact of germline filtering was evaluated.

\section{Results}

Sixteen samples demonstrated low interlaboratory and interpanel psTMB variation with $87.7 \%$ of pairwise comparisons showing a Spearman's $\rho>0.6$. A wesTMB cutpoint of 199 missense mutations projected to psTMB cutpoints between 7.8 and 12.6 muts/Mbp; the corresponding psTMB and wesTMB classifications agreed in $74.9 \%$ of cases. For threetier classification with cutpoints of 100 and 300 mutations, agreement was observed in $76.7 \%$, weak misclassification in $21.8 \%$, and strong misclassification in $1.5 \%$ of cases. Confounders of psTMB estimation included fixation artifacts, DNA input, sequencing depth, genome coverage, and variant allele frequency cutpoints.

\section{Conclusions}

This study provides real-world evidence that all evaluated panels can be used to estimate TMB in a routine diagnostic setting and identifies important parameters for reliable tissue TMB assessment that require careful control. As complex/composite biomarkers beyond TMB are likely playing an increasing role in therapy prediction, the efforts by QuIP and FoCR also delineate a general framework and blueprint for the evaluation of such assays. 
Keywords: tumor mutational burden, TMB, lung cancer, gene panel, sequencing, immuno-oncology, quality assurance

\section{Introduction}

Immune checkpoint inhibitors (ICls) have greatly expanded therapeutic options in oncology. ${ }^{1}$ While many clinical trials have shown strong clinical responses across various tumor types, evidence is increasing that even in generally responsive tumor entities many tumors are resistant at baseline or develop resistance to ICls, e.g., by immunoediting. ${ }^{2}$ Moreover, adverse events associated with ICls have been noted, particularly with combinatorial regimens that target cytotoxic T-lymphocyte-associated protein 4 (CTLA-4) in addition to programmed cell death protein 1 (PD-1) or programmed death-ligand 1 (PDL1). ${ }^{3}$ Collectively, these observations argue for a sophisticated biomarker approach that reflects the interplay between the host's immune system and the cancer cells and is able to reliably separate likely responders from nonresponders.

To date, two predictive $\mathrm{ICl}$-specific biomarkers have been approved in certain cancer types: (i) PD-L1, assessed by immunohistochemistry (IHC) with a wide range of different scoring systems and cutpoints depending on cancer type-specific trial results, and (ii) highlevel microsatellite instability (MSI-H) or mismatch repair deficiency, assessed by either polymerase chain reaction or $\mathrm{IHC}^{4,5}$ Whereas the former approach measures a continuous variable that serves as an approximation for T-cell anergy or tumor cells escaping immune response, the latter identifies a subgroup of cancers with a high mutational burden and thus increased neoantigen load, which likely results in a higher propensity of immune cell-mediated tumor cell killing. 
However, many cancer types, including non-small cell lung cancer (NSCLC), do not harbor deleterious mutations in one of the DNA mismatch repair genes but show increased tumor mutational burden (TMB) associated with higher loads of neoantigens which is caused by DNA damage through external noxae (e.g., UV light, smoking) or deleterious mutations affecting other DNA repair genes. ${ }^{6}$

Although clinical trials assessing the utility of TMB prospectively are ongoing, many retrospective analyses of individual patient cohorts and clinical trials have demonstrated that TMB can be successfully used for patient stratification. Initial seminal studies employed whole exome sequencing (WES) to measure TMB. ${ }^{7-10}$ Because this approach has several limitations, including sample requirements, necessity for concurrent germline sequencing, extensive laboratory capacity for diagnostic application, and economic constraints in consideration of a diagnostic outreach setting, gene panels were designed and used to estimate TMB values, primarily in formalin-fixed and paraffin-embedded (FFPE) tissue and, more recently, in cell-free circulating tumor DNA. ${ }^{11-13}$ Such assays have been successfully used under controlled trial conditions or at specific academic cancer centers. However, a detailed evaluation of the overall performance of commercially available sequencing panels that can be used as laboratory-developed tests (LDTs) as well as of the parameters affecting its diagnostic applicability is missing.

To address this important issue, we present the results of the multi-institutional Quality in Pathology (QuIP) study on a comparative assessment of TMB estimated by gene panel sequencing (psTMB) from 11 different institutes of pathology and four industrial laboratories. Analyzing 20 different FFPE cancer samples from routine diagnostics that reflect the full continuum of TMB, as measured by WES (wesTMB), provides real-world data on six different targeted gene panels designed for TMB estimation: Oncomine Tumor 
Mutational Load Assay (OTML; Thermo Fisher Scientific, Waltham, MA), QIAseq TMB panel (QIAseq; QIAGEN GmbH, Hilden, Germany), NEOplus RUO assay (NEOplus; NEO New Oncology, Cologne, Germany), TruSight Oncology 500 panel (TSO500; Illumina, San Diego, CA), a custom-designed academic panel (ACADEMIC; Agilent, Santa Clara, CA), and the FoundationOne assay (F1; Foundation Medicine, Cambridge, MA). Together with the efforts led by the Friends of Cancer Research (FoCR), ${ }^{14}$ this study sets the basis for harmonization of panel-based TMB measurement and supports implementation of TMB in routine diagnostic laboratories.

\section{Materials and Methods}

\section{Samples}

All patients provided written informed consent under an institutional review boardapproved protocol, and the study was conducted in accordance with the Declaration of Helsinki. FFPE tissue specimens of 10 lung adenocarcinoma (LUAD), seven head and neck squamous cell carcinoma (HNSC), and three colon adenocarcinoma (COAD) were prepared and diagnosed at the Institute of Pathology Heidelberg, Germany. See Suppl. 1 for further detailed sample information. Only one block per tumor was selected and consecutive sections were used for DNA extraction by the different laboratories. Tumor content was controlled using HE stained slides on the first and last section to ensure homogeneity throughout the slices.

\section{Library Preparation and Sequencing}

Protocols for the six applied panel-sequencing approaches (OTML, QIAseq, NEOplus, TSO500, ACADEMIC, and F1), as well as for WES, are detailed in the Supplementary Appendix (Suppl. 2). All assays were performed according to the manufacturers' protocols if not specified otherwise. 


\section{Data Analysis and Visualization}

Data analysis and visualization were performed using the statistical programming language $\mathrm{R}^{15}$ Levels of psTMB were visualized as boxplots and as heatmaps including hierarchical clustering of experiments (Manhattan distance, average linkage clustering). Spearman correlations $(\rho)$ and Pearson correlations (R) of psTMB were calculated between pairs of experiments, clustered (Euclidean distance, average linkage clustering), and visualized as heatmaps. Error bars were plotted using the function plotCl from the $\mathrm{R}$ package gplots. Violin plots were generated using the $\mathrm{R}$ package vioplot.

Linear models without intercept were fitted to psTMB levels with wesTMB levels. Measurement of psTMB is influenced by different factors. Although misclassification of germline mutations as somatic mutations is independent of the TMB level, other factors, including the subsampling error caused by interrogation of only a limited part of the coding sequence (CDS), increase with a higher TMB. ${ }^{16}$ Because the exact shape of the mathematical dependence of the TMB error on the level of the TMB is not known, linear models were fitted in two different ways: (i) standard linear regression (least square regression, LS) corresponding to constant error contributions, and (ii) weighted linear regression (weighted least squares, WLS) with weights equal to the reciprocal of TMB taking into account heteroscedasticity. The shape of the weights used in the WLS model reflects the mathematical law for the variation of psTMB that we recently uncovered and described-a linear increase of the variation of psTMB proportional to the level of TMB. ${ }^{16}$ 


\section{Results}

\section{Study Outline}

In this study (Fig. 1), FFPE tissue samples of 20 tumors (Suppl. 1) with existing matched WES data were analyzed using four commercial panel-sequencing TMB assays (Suppl. 2). Each assay was run by four different pathology laboratories as well as by a reference laboratory of the panel provider on all samples. Additionally, three pathology laboratories tested the ACADEMIC assay, and all samples were analyzed using the $\mathrm{F} 1$ assay. The analyzed study cohort was selected to represent the full spectrum of TMB values as characterized by The Cancer Genome Atlas for LUAD, HNSC, and COAD, but has a higher proportion of tumors with an intermediate TMB (100 to 300 mutations, Suppl. 3). In total, panel sequencing and psTMB measurement was successful in $97.3 \%$ (467 of 480 ) of the performed analyses.

\section{TMB Levels and Correlations}

Measurements of psTMB in the 20 tumor-tissue samples ranged between 0 and 244 mutations per megabase pair (muts/Mbp) with a median of 9.2 muts/Mbp (Fig. 2A). With respect to interlaboratory and interpanel variance, four of the tumor samples (T4, T7, T13, and T15) stood out by showing a larger interquartile range of psTMB compared with the remaining samples. This was mainly due to unfavorable preanalytic quality parameters (degraded DNA or low tumor cellularity, Fig. 2A). Two samples (T4 and T15) showed a large interlaboratory variance of psTMB when each of the panels was analyzed separately, whereas this was not the case for the two other tumor samples (T7 and T13), where interpanel variance was an important confounder (Suppl. 4).

In a heatmap including hierarchical clustering of the psTMB levels, data readouts based on the same sequencing panel often clustered together, indicating independence from the 
operating laboratory (Fig. 2B). Between most of the sequencing results, moderate to strong pairwise correlations of psTMB measurements were observed: of all pairwise Spearman correlations, $65.9 \%$ were $\geq 0.7,87.7 \%$ were $\geq 0.6$, and $95.7 \%$ were $\geq 0.5$ (Fig. 2C). In the study cohort, the strength of Pearson correlations was dependent on the inclusion or exclusion of a single sample (T16, POLE-mutated colorectal carcinoma) that had a very high TMB (>100 muts/Mbp, Suppl. 5). Hence, the Spearman correlation was more suitable approach for the measurement of the psTMB correlations than the Pearson method.

\section{Bridging from psTMB to wesTMB}

Linear regression models were fitted for bridging from psTMB to wesTMB (Fig. 3). To this end, we performed standard linear regression (LS), but also weighted linear regression (WLS, see Materials and Methods for details) for each of the panels tested in the study. Bridging factors (BFs) for transformation of psTMB to wesTMB were calculated as reciprocals of the regression slopes (Suppl. 6). For most of the assays, the BF determined by WLS was very close to the BF determined by LS. However, for the ACADEMIC assay, the WLS BF was slightly lower than the LS BF (17.7 vs. 19.8), whereas it was considerably lower for the QIAseq assay (15.8 vs. 25.6).

A clinically relevant psTMB cutpoint of 10 muts/Mbp in NSCLC was established in the CheckMate 568 study using the F1 panel, evaluated in the CheckMate 227 study, and bridged to a wesTMB cutpoint of 199 mutations using data from the CheckMate 026 study. ${ }^{17-19}$ Based on these findings, we calculated psTMB cutpoints corresponding to 199 mutations for each of the investigated assays (Suppl. 6). For most of the psTMB assays, the calculated cutpoints were consistently in the range of 9.4 to 11.5 muts/Mbp. There 
were two exceptions: considerably different cutpoints were obtained for the OTML assay (LS: 7.8 muts/Mbp, WLS: 7.9 muts/Mbp) and for the QIAseq assay (LS: 7.8 muts/Mbp, WLS: 12.6 muts/Mbp).

\section{TMB Classification}

Next, we evaluated a two-tier in comparison to a three-tier system for TMB classification (Fig. 4) following a recent indication to improve the misclassification ratio. For the two-tier approach, a dichotomization into "low TMB" and "high TMB" was conducted using a wesTMB cutpoint of 199 mutations. The three-tier classification classified TMB as "low" (<100 mutations), "intermediate" (100 to 300 mutations) and "high" ( $\geq 300$ mutations). Classification with alternative cutpoints (150 and 250 mutations) is shown in Suppl. 7. For each of the panel sequencing platforms, psTMB values were converted to wesTMB values using the BFs obtained by WLS regression. Altogether (20 samples $\times 24$ experiments), we observed an agreement between psTMB and wesTMB classifications in $74.9 \%$ of the cases using the two-tier approach. For the three-tier approach, a "strong misclassification" was defined by a high TMB tumor classified as low TMB or vice versa (difference spanning two tiers), whereas a misclassification by a single tier (e.g., intermediate TMB to low TMB) was termed "weak misclassification." Here, we observed an agreement in $76.7 \%$ of cases, compared with a weak and strong misclassification in $21.8 \%$ and $1.5 \%$ of the cases, respectively. Of note, strong misclassification occurred only for a single tumor sample (T4) that was classified as low TMB by WES but as high TMB in seven psTMB assays and was not analyzable in five psTMB approaches. Assessment of this tumor (LUAD) was priori expected to be challenging due to highly degraded DNA. 
TMB classifications using BFs determined either by WLS or LS regression were similar, as LS regression resulted in $74.3 \%$ agreement for two-tier classification as well as $75.0 \%$ agreement, $23.1 \%$ weak misclassifications, and $1.9 \%$ strong misclassifications for three tier classification.

\section{Interlaboratory comparison of the identified variants}

In-depth analysis of called variants included in the calculation of TMB identified key factors that influence precise psTMB estimation from FFPE tissue (Fig. 5). A sequencing approach without an application for PCR duplicate removal, known as deduplication, has a higher probability of erroneous calling of $C>T / G>A$ fixation artifacts and subsequent overestimation of psTMB, especially in highly fragmented, low-quality DNA samples. Methods for deduplication include specialized software solutions as well as the use of unique molecular identifiers (UMls, or molecular barcodes).

False-positive variants in the generated data set were identified by a side-to-side comparison of all variants identified by the different laboratories using the same panel. Variants were classified into non-reproducible variants (detected by a single laboratory), partially reproducible variants (detected by more than one laboratory, but not by all laboratories) and fully reproducible variants (detected by all laboratories). Variant allele frequencies (VAFs) were considerably lower for variants with low degrees of interlaboratory reproducibility and many of the non-reproducible variants had VAFs close to the VAF cutpoint (Fig. 5A). Thus, low frequency variants close to the VAF cutpoint contributed considerably to psTMB variation. To minimize the rate of false-positive calls, specific thresholds for VAF were used for each panel according to the assay provider: VAF $\geq 10 \%$ was applied for the OTML and NEOplus panels and VAF $\geq 5 \%$ for the remaining panels. The number of non-reproducible variants was considerably higher for the OTML 
assay (3497 variants), which did not include deduplication, compared with the other assays (QIAseq: 1055; NEOplus: 94; TSO500: 70; ACADEMIC: 691). In addition, as illustrated in Fig. $5 B$, the ratio of $C>T / G>A$ transitions was significantly higher for nonreproducible variants (red pie charts) detected by the OTML panel (86\%) compared with the other panels $(22 \%-42 \%)$, and also compared with the ratio of $C>T / G>A$ of variants that were detected by all laboratories (gray pie charts). These data identify paraffin fixation artifacts and resulting $\mathrm{C}>\mathrm{T} / \mathrm{G}>\mathrm{A}$ transitions as important parameters contributing to falsepositive variant detection for assays that do not employ deduplication.

False-negative calls (defined here as mutations called by all but one laboratory) can be connected to insufficient depth of coverage at the respective positions. Because the pipelines for capture-based fragment libraries typically include deduplication and UMI filtering, the depth of coverage directly correlates with the amount of DNA input, as shown representatively for the TSO500 panel in Fig. 5C. Here, the median exon coverage that could be analyzed was significantly higher in lab 1 using 80 ng as DNA input compared with $40 \mathrm{ng}$ that was used for the other TSO500 approaches (labs 2, 7, 11, and Illumina). Further, the amount of DNA input had a strong impact on the average size of the covered sequencing region (Fig. 5C, middle). While the maximum covered coding region size of 1.28 Mbp was reached for all samples using $80 \mathrm{ng}$ (lab 1), lower DNA input resulted in significant $(p<0.01)$ lower covered coding region sizes, which were larger than $1.0 \mathrm{Mbp}$ in $35 \%$ to $100 \%$ of the analyzed samples. To enhance specificity, only mutations with minimum coverage of $100 x$ were included in the psTMB calculation. Therefore, and connected to the lower coverage, we observed a higher rate of false-negative variants in analyses using 40 ng DNA (labs 2, 7, 11, and Illumina) compared with 80 ng (Fig. 5C, 
right). Similar findings were seen for $100 \mathrm{ng}$ vs. $200 \mathrm{ng}$ DNA input using the NEOplus assay (data not shown).

\section{Germline Mutation Filtering}

Germline mutation filtering is an important step in the calculation of psTMB because only the tumor's somatic mutations are relevant for recognition by the immune system. In the absence of sequencing of paired-normal tissue/blood samples in most diagnostic scenarios, germline mutation filtering needs to be performed in silico. For all assays evaluated in the current TMB harmonization study, the bioinformatic pipelines include a step of negative filtering for entries in single nucleotide polymorphism (SNP) databases such as gnomAD, ExAC, and dbSNP. Additionally, some of the pipelines include further steps; for example, filtering by algorithms specifically designed to distinguish somatic vs. germline mutations such as somatic-germline zygosity (SGZ) or filtering with respect to the mutations detected by panel sequencing of reference cohorts of normal samples (e.g., NEOplus, ACADEMIC panel). ${ }^{20}$ We evaluated the performance of filtering using SNP databases for the LUAD samples ( $n=10$; Suppl. 8 ). Variants detected by WES in matched blood samples were used as a reference. The sensitivity for classifying mutations as somatic was $87 \%, 90 \%$, and $79 \%$, with corresponding positive predictive values (PPVs) of $90 \%, 90 \%$, and $91 \%$ when using gnomAD, ExAC, and dbSNP for filtering (pooled analysis of the 10 tumor samples). Filtering out only common SNPs (minor allele frequency [MAF] > 0.001 in gnomAD) increased sensitivity to $98 \%$, but decreased PPV to $81 \%$.

While germline mutation filtering using gnomAD and ExAC performed well, rs-filtering (dbSNP) appeared to be too stringent. Restriction of filtering to common SNPs considerably decreased the number of false negatives, but increased the number of false 
positives. Additional filters that are implemented in the panel-specific bioinformatic pipelines, such as SGZ algorithm or the TSO500 "proxy filter" (Suppl. 9), can further improve germline mutation filtering. 


\section{Discussion}

Tumor vs. matched blood WES was used in many initial clinical I-O studies and may be considered a reference standard for TMB assessment. However, clinical implementation of WES-based TMB assessment may be impractical, considering the financial costs and the limited availability of appropriately-preserved samples or quality DNA, and matched normal samples for germline sequencing. Gene panel assays offer a number of economical and practical advantages for clinical assessment of patient samples, including increased sequencing depth, in silico germline subtraction (negating the requirement for matched samples), and concurrent evaluation of actionable mutations.

The QuIP study provides a thorough analysis of real-world performances of six select TMB panels. Using real-world diagnostic FFPE samples, which included different types of challenging cases with poor DNA quality, heavy fixation artifacts, or low tumor cellularity, our results demonstrate that in principle all assays tested in this study were able to estimate TMB values and could be applied in a diagnostic setting.

The effect of panel size and coverage on the accuracy of psTMB assessment have been previously studied using in silico simulations of gene panels derived from WES data. ${ }^{16,21}$ The gene panels used in the LDTs covered at least $1 \mathrm{Mbp}$ of the CDS, which was shown to be essential for valid panel-based TMB assessment. ${ }^{21}$ However, even with these large panels, variability of the TMB score can be expected because psTMB measurement has a probabilistic nature: the overall TMB is extrapolated by investigating only a fraction (about $1 / 30$ ) of the exome. Simulating five commercial panels in WES data, only $17 \%-28 \%$ of additional error occurred on top of the unavoidable probabilistic error demonstrating that sufficient panel size is more critical than the particular localization of the panel in the exome. $^{16}$

There is a multitude of other wet-lab parameters, ranging from biological factors (e.g., tumor heterogeneity) and preanalytics (e.g., DNA quality) to sequencing (e.g., coverage) 
as well as bioinformatics parameters (e.g., germline subtraction) that can influence TMB scores. $^{22-25}$ Hence, as expected, absolute TMB values slightly varied. This scenario is not unknown to pathology in general and immune oncology response prediction in particular: just as for TMB, the established PD-L1 IHC assay for NSCLC quantifies a continuous variable in tumor cells ranging from $0 \%$ to $100 \%$ PD-L1 expressing cells, and several parameters, such as tumor heterogeneity and fixation, are known to influence PD-L1 scores. ${ }^{4,26}$ Just as with PD-L1, for clinical purposes TMB as a continuous measure must be categorized. In our approach we stratified samples into one of three groups, which categorized the continuum of TMB ranging from 0 to $>200$ muts/Mbp: low, intermediate, and high TMB, according to a concept proposed by us recently. ${ }^{16}$ In contrast to a two-tier system with one defined cutoff, this concept allows for a definition of a certain "intermediate" grey zone of TMB measurements in an area around the currently proposed clinical cutpoint. Using cutpoints of 100 mutations (corresponding to approximately 5 muts/Mbp) and 300 mutations (corresponding to approximately 15 muts/Mbp), strong misclassifications occurred only for a single tumor sample (T4), a case that was particularly challenging because of poor DNA quality, which would justify to decline analysis in a clinical setting. Misclassification of other highly degraded samples (T12 and T19) or critical cases with a low tumor-cell content (T15), MSI-H status (T13 and T15), or a loss-of-function mutation in POLE (T16) was prevented using the three-tier system instead of the two-tier system.

We observed an overall low influence of the specific laboratory performing the analysis; data generated by the industrial partners for their specific panel were in the range of the respective $\mathrm{TMB}$ scores determined by the hospital laboratories. Moreover, we found that most panels showed moderate to strong ( $\rho=0.64$ to 0.84$)$ correlations with TMB measured by WES. 
Our study also showed that germline subtraction using bioinformatic pipelines can be used to identify likely somatic variants in the probabilistic setting of psTMB measurement. However, as shown by us recently, incorrect filtering can influence TMB scores in individual cases, and future studies are warranted to further investigate the influence of in silico vs. blood-based subtraction of germline events. ${ }^{24,27}$ As current germline variant databases are biased toward e.g. Caucasian populations, ethnicity-related aspects require careful analysis in this context.

We identified assay-independent and assay-specific parameters (Fig. 6) that will require careful control when psTMB is implemented in routine diagnostics. Of these, the effects of tumor-cell content, DNA input and coverage are most critical in order to prevent the miss of mutations which would result in too low psTMB scores. Another important aspect are deamination artifacts ( $\mathrm{C}>\mathrm{T}$ transitions) created by formalin fixation, which can be diagnostically challenging when left uncontrolled. In this regard DNA amplification during panel sequencing can be critical as overamplification of artefacts or additional errors during replication can occur leading to false positive mutation calls and subsequently to overestimated psTMB scores. This issue can be compensated by setting an appropriate limit of detection (LOD) for the allelic frequency and especially by applying in silico and/or technical (molecular barcodes) approaches for deduplication (removal of PCR duplicates). In the present data set a LOD of $5 \%$ in combination with deduplication yielded reliable mutation calling, and eventually TMB values. Hybrid-capture based target enrichment was favorable in this context. In panels without deduplication, deamination artifacts may be controlled by increasing the LOD to e.g. $10 \%$, rendering cases with low tumor cellularity challenging due to the impaired sensitivity. Recent reports indicate that the application of uracil-DNA glycosylase (UDG), an enzyme selectively digesting uracil-containing nucleic 
acids, can reduce deamination artifacts, when assessing TMB in FFPE samples using assays without a deduplication approach. ${ }^{28-30}$ However, the effect of this approach was not tested in this study.

We also calculated bridging factors to convert psTMB to wesTMB for the assessed panels. Although future studies exploring larger sample sets will likely improve this analysis, we believe that the data shown here provide a strong and sound basis that will facilitate the comparison of TMB values obtained by different panels.

A limitation of our study is the limited number of cases as well as the use of three different cancer types. The latter selection was influenced by (i) a case mix that reflects the continuum of TMB, (ii) avoiding results bias due to a single cancer type, (iii) tissue availability for the entire study and all partners, and (iv) availability of corresponding WES data. Since the predictive power of TMB is currently being tested in many immunooncology trials across various cancer types and as our study is primarily aimed at investigating the ability of panels to measure $T M B$, we believe that these points do not interfere with our results and conclusions.

In summary, the QuIP study provides real-world evidence that all panels tested in this study can be used to estimate TMB by panel sequencing from FFPE samples in a routine diagnostic setting. However, this study has identified a number of critical parameters, including sample fixation, DNA input, sequencing depth, genome coverage, and variant allele frequency cutpoints, that may confound psTMB estimation and require careful control to achieve successful and reliable psTMB analysis. Beyond TMB, in conjunction with efforts by FoCR, this study provides a blueprint and framework for the systematic 
analysis of complex/composite predictive biomarkers, which will likely play an increasing role in guiding oncological therapy. 


\section{Acknowledgments}

We thank all teams of the participating institutions for their contributions, and the QulP team for excellent administration. We also thank the entire team of the Center for Molecular Pathology (CMP) at the Institute of Pathology Heidelberg (IPH) for expert technical assistance and fruitful discussions. The support of the HIPO program and GPCF (both DKFZ) are gratefully acknowledged. Editorial assistance was provided by Stuart Rulten, PhD, and Jay Rathi, MA, of Spark Medica Inc., funded by Bristol-Myers Squibb. We thank David Fabrizio for fruitful discussions related to this project. We thank Sandra Siesing for expert editorial assistance and overall handling of the manuscript.

\section{Conflicts of Interests}

The study was partly sponsored by Bristol-Myers Squibb, Illumina, MSD, FMI, Neo New Oncology, QIAGEN, Roche, and Thermo Fisher Scientific.

AS: Advisory board honoraria from: AstraZeneca, Bayer, BMS, Illumina, Novartis, Seattle Genomics, Takeda, ThermoFisher. Speaker's honoraria: AstraZeneca, Bayer, BMS, Illumina, MSD, Novartis, Roche, Seattle Genomics, Takeda, ThermoFisher. Research funding: Chugai, BMS, Bayer

VE: personal fees from Thermo Fisher, personal fees from Astra Zeneca

JB: has nothing to disclose.

SMB: QuIP, during the conduct of the study. Personal fees from BMS, personal fees from Roche, personal fees from Novartis, personal fees from Pfizer, personal fees from Astra Zeneca, personal fees from Bayer, non-financial support from Illumina, non-financial support from Janssen

WD: Stockholding Healthineers.

NP: has nothing to disclose.

US: has nothing to disclose.

$\mathrm{MH}$ : has nothing to disclose.

$\mathrm{JA}$ : has nothing to disclose.

$\mathrm{SH}$ : Dr. Herold

MZ: Grants from F. Hoffmann-La Roche AG, Basel, $\mathrm{CH}$, during the conduct of the study; grants from F. Hoffmann-La Roche AG, Basel CH outside the submitted work.

DK: personal fees from Pfizer Pharma GmbH

JM: personal fees from BMS, personal fees from

DMM: has nothing to disclose.

MSt: has nothing to disclose.

JA: has nothing to disclose.

KZ: Dr. Zaoui

MSch: has nothing to disclose.

HG: has nothing to disclose.

$\mathrm{DH}$ : personal fees from Bayer, personal fees from BMS, personal fees from Roche 
MT: other from MSD, other from BMS

ME: has nothing to disclose.

HM: grants from Roche, during the conduct of the study. Grants from Roche, personal fees from

Roche, outside the submitted work.

TK: has nothing to disclose.

RB: Professor Büttner

PS: grants from QuIP, during the conduct of the study; grants and personal fees from BMS, grants and personal fees from MSD, grants and personal fees from Roche, grants and personal fees from AstraZeneca, grants and personal fees from Novartis, personal fees from Chugai, personal fees from AbbVie, grants from Sanofi-Aventis, personal fees from Ipsen, grants and personal fees from Pfizer, grants from Illumina, grants from Thermo Fisher, outside the submitted work.

ER: has nothing to disclose.

AJ: other from QuIP GmbH, personal fees and non-financial support from BMS, during the conduct of the study. Personal fees and non-financial support from Amgen, personal fees and non-financial support from Boehringer Ingelheim, personal fees and non-financial support from Novartis, personal fees and non-financial support from Bayer, personal fees and non-financial support from Merck Serono, personal fees and non-financial support from Roche Pharma, personal fees and non-financial support from Biocartis, personal fees and non-financial support from Merck Sharp Dome (MSD), personal fees and non-financial support from ThermoFisher, outside the submitted work.

FH: non-financial support from Illumina, non-financial support from Qiagen, during the conduct of the study: Grants, personal fees and non-financial support from Illumina, personal fees and nonfinancial support from Qiagen, personal fees from BMS, personal fees from Novartis, personal fees from Merck, personal fees from Astra Zeneca, personal fees from Pfizer, personal fees from Bayer, outside the submitted work.

LT: non-financial support from Illumina, Inc, non-financial support from QIAGEN, non-financial support from Agilent Technologies, during the conduct of the study.

WW: personal fees from Roche, MSD, BMS, AstraZeneca, Pfizer, Merck, Lilly, Boehringer, Novartis, Takeda, Amgen, Astellas and grants from Roche, MSD, BMS, Bruker outside the submitted work.

MD: Professor Dietel

SF: personal fees from Amgen, grants from AstraZeneca, personal fees from Eli Lilly, grants from Pfizer, grants from PharmaMar, personal fees from Roche, personal fees from Bayer, outside the submitted work.

GB: Dr. Baretton has nothing to disclose.

CW: Professor Wickenhauser

\section{Author Contributions}

Conceived and designed the study: MD, RB, SMB, FH, WW, AJ, JM, PS, MH, TK, HM,

DK, JB, VE, AS

Provided samples: DH, SF, PS, HG, AS, VE

Sequenced and analyzed cases: AS, VE, JB, SMB, DK, WD, NP, US, MH, SH, JA, MZ,

LT, ER, MS, HG, SF, JA, DH, GB, CW, MT, ME, HM, TK, RB, OS, AJ, FH, WW, MD

Statistical analyses: JB, ER, VE, DK, AS

Manuscript draft: AS, DK, VE, JB, PS, MD 
Contribution to writing: all authors

All authors read and approved the manuscript. 


\section{Figure Legends}

Figure 1. Outline of the QuIP TMB harmonization study. In this comparative study, FFPE tissue samples from 20 tumors were analyzed using four commercial panel-sequencing TMB assays. Each assay was tested by four independent pathology laboratories using all 20 samples as well as by a reference laboratory of the panel provider. Additionally, all samples were analyzed using an "academic" assay in three hospital laboratories, and by applying the F1 assay. The study cohort consisted of 20 samples from patients with LUAD $(n=10)$, HNSCC $(n=7)$, and COAD $(n=3)$. For all tumors, wesTMB using fresh-frozen tumor tissue samples and paired-blood samples was available. ACADEMIC, customdesigned academic panel; COAD, colon adenocarcinoma; F1, FoundationOne assay; FFPE, formalin-fixed and paraffin-embedded; HNSCC, head and neck squamous cell carcinoma; LUAD, lung adenocarcinoma; NEOplus, NEOplus RUO assay; OTML, Oncomine Tumor Mutational Load Assay; psTMB, TMB assessed by gene panel sequencing; QIAseq, QIAseq TMB panel; TMB, tumor mutational burden; TSO500, TruSight Oncology 500 panel; WES, whole exome sequencing; wesTMB, TMB assessed by WES.

Figure 2. (A) Overview of the generated psTMB estimates with tumors ordered by increasing wesTMB levels. Applying a three-tier classification system, four tumors (T1-T4) were classified as TMB low ( $<100$ missense mutations), eleven tumors were classified as TMB intermediate (100 to 300 missense mutations), and five tumors (T5-T7, T13, and T16) were classified as TMB high ( $\geq 300$ missense mutations). Four samples stood out by high interquartile ranges and are marked by red IDs. Preanalytic quality parameters were unfavorable for three of these samples (T15: low tumor cellularity; T4 and T13: degraded DNA). (B) Heatmap of psTMB levels. Red colors: psTMB level $>10$ muts/Mbp. Green 
colors: psTMB level $<10$ muts/Mbp. White: insufficient DNA quality. (C) Spearman correlations between psTMB and wesTMB levels in the study cohort. ACADEMIC, customdesigned academic panel; F1, FoundationOne assay; muts/Mbp, mutations per megabase pair; NEOplus, NEOplus RUO assay; OTML, Oncomine Tumor Mutational Load Assay; psTMB, TMB assessed by gene panel sequencing; QIAseq, QIAseq TMB panel; TMB, tumor mutational burden; TSO500, TruSight Oncology 500 panel; WES, whole exome sequencing; wesTMB, TMB assessed by WES.

Figure 3. Calibration of TMB measured by psTMB against wesTMB. Linear fits using LS and WLS regression. (A) Overview plots showing all psTMB and wesTMB measurements. (B) Zoom-ins to the intervals $[0,650]$ of wesTMB and $[0,65]$ of psTMB. The intercepts in the linear regression models were set to zero. ACADEMIC, custom-designed academic panel; F1, FoundationOne assay; LS, least squares; muts/Mbp, mutations per megabase pair; NEOplus, NEOplus RUO assay; OTML, Oncomine Tumor Mutational Load Assay; psTMB, TMB assessed by gene panel sequencing; QIAseq, QIAseq TMB panel; TMB, tumor mutational burden; TSO500, TruSight Oncology 500 panel; WES, whole exome sequencing; wesTMB, TMB assessed by WES; WLS, weighted least squares.

Figure 4. TMB classification by panel sequencing compared with TMB classification by WES. Measurements of psTMB were converted to wesTMB using the bridging factors in Suppl. 6. (A) Two-tier classification using the cutpoint of 199 mutations. Misclassifications: 25.1\%. (B) Three-tier classification using the cutpoints of 100 and 300 mutations. Red: high TMB; yellow: intermediate TMB; green: low TMB. Strong misclassifications (= misclassifications mixing TMB high and TMB low cases): $1.5 \%$. Weak misclassifications (= misclassifications mixing intermediate TMB cases with TMB high or TMB low cases): 
21.8\%. ACADEMIC, custom-designed academic panel; F1, FoundationOne assay; NEOplus, NEOplus RUO assay; OTML, Oncomine Tumor Mutational Load Assay; psTMB, TMB assessed by gene panel sequencing; QIAseq, QIAseq TMB panel; TMB, tumor mutational burden; TSO500, TruSight Oncology 500 panel; WES, whole exome sequencing; wesTMB, TMB assessed by WES.

Figure 5. Interlaboratory reproducibility of the detected mutations (pooled analysis of 20 samples). (A) Distribution of VAFs in dependence of the number of laboratories that detected the mutation. (B) Mutation type ( $\mathrm{C}>\mathrm{T} / \mathrm{G}>\mathrm{A}$ or other) of the mutations detected by a single lab. (C) Impact on DNA input is representatively shown for the TSO500 panel. DNA input: 80 ng (lab 1), 40 ng (labs 2, 7, 11, and Illumina). Left: Median exon coverage for each sample; the number on top gives the percentage of cases with a median exon coverage of more than 150x. Middle: Covered coding region size for each sample. The number on top gives the percentage of cases with a covered coding region of more than 1.0 Mbp. Right: Coverage of mutations not-called by a single lab (false negatives). ACADEMIC, custom-designed academic panel; NEOplus, NEOplus RUO assay; OTML, Oncomine Tumor Mutational Load Assay; QIAseq, QIAseq TMB panel; TSO500, TruSight Oncology 500 panel; VAF, variant allele frequency.

Figure 6. Schematic representation of assay-independent and assay-specific parameters influencing the accuracy of psTMB scores. Lower lane: Four representative samples showing the effect of deduplication strategies (\#2), insufficient sample material (\#3) or low tumor purity (\#4) on DNA input, coverage, covered coding sequence, variant calling, and the resulting psTMB scores. Red arrow pointing down indicates false negative effect. Red 
arrow pointing up indicates false positive effect. psTMB, TMB assessed by gene panel sequencing; wesTMB, TMB assessed by WES. 


\section{References}

1. Havel JJ, Chowell D, Chan TA. The evolving landscape of biomarkers for checkpoint inhibitor immunotherapy. Nat Rev Cancer. 2019;19(3):133-150.

2. Jenkins RW, Barbie DA, Flaherty KT. Mechanisms of resistance to immune checkpoint inhibitors. Br J Cancer. 2018;118(1):9-16.

3. Brahmer JR, Lacchetti C, Schneider BJ, et al. Management of immune-related adverse events in patients treated with immune checkpoint inhibitor therapy: American Society of Clinical Oncology Clinical Practice Guideline. J Clin Oncol. 2018;36(17):1714-1768.

4. Buttner R, Gosney JR, Skov BG, et al. Programmed death-ligand 1 immunohistochemistry testing: a review of analytical assays and clinical implementation in non-small-cell lung cancer. J Clin Oncol. 2017;35(34):38673876.

5. Le DT, Uram JN, Wang $\mathrm{H}$, et al. PD-1 blockade in tumors with mismatch-repair deficiency. N Engl J Med. 2015;372(26):2509-2520.

6. Campbell BB, Light N, Fabrizio D, et al. Comprehensive analysis of hypermutation in human cancer. Cell. 2017;171(5):1042-1056.

7. Carbone DP, Reck M, Paz-Ares L, et al. First-line nivolumab in stage IV or recurrent non-small-cell lung cancer. N Engl J Med. 2017;376(25):2415-2426. 
8. Rizvi NA, Hellmann MD, Snyder A, et al. Cancer immunology. Mutational landscape determines sensitivity to PD-1 blockade in non-small cell lung cancer. Science. 2015;348(6230):124-128.

9. Snyder A, Makarov V, Merghoub T, et al. Genetic basis for clinical response to CTLA-4 blockade in melanoma. N Engl J Med. 2014;371(23):2189-2199.

10. Van Allen EM, Miao D, Schilling B, et al. Genomic correlates of response to CTLA-4 blockade in metastatic melanoma. Science. 2015;350(6257):207-211.

11. Chalmers ZR, Connelly CF, Fabrizio D, et al. Analysis of 100,000 human cancer genomes reveals the landscape of tumor mutational burden. Genome medicine. 2017;9(1):34.

12. Gandara D, Paul S, Kowanetz M, et al. Blood-based tumor mutational burden as a predictor of clinical benefit in non-small-cell lung cancer patients treated with atezolizumab. Nat Med. 2018;24(9):1441-1448.

13. Zehir A, Benayed R, Shah RH, et al. Mutational landscape of metastatic cancer revealed from prospective clinical sequencing of 10,000 patients. Nat Med. 2017;23(6):703-713.

14. Merino DM, McShane L, Butler M, et al. TMB standardization by alignment to reference standards: Phase II of the Friends of Cancer Research TMB Harmonization Project. J Clin Oncol. 2019;37(15_suppl): Abstract 2624. 
15. R Development Core Team. R: A language and environment for statistical computing. 2010; http://www.r-project.org. Accessed September 19, 2019.

16. Budczies J, Allgauer M, Litchfield K, et al. Optimizing panel-based tumor mutational burden (TMB) measurement. Ann Oncol. 2019;30(9):1496-1506.

17. Hellmann MD, Ciuleanu TE, Pluzanski A, et al. Nivolumab plus Ipilimumab in Lung Cancer with a High Tumor Mutational Burden. N Engl J Med. 2018;378(22):20932104.

18. Hellmann MD, Paz-Ares L, Bernabe Caro R, et al. Nivolumab plus Ipilimumab in Advanced Non-Small-Cell Lung Cancer. N Engl J Med. 2019;381(21):2020-2031.

19. Ready N, Hellmann MD, Awad MM, et al. First-line nivolumab plus ipilimumab in advanced non-small-cell lung cancer (CheckMate 568): outcomes by programmed death ligand 1 and tumor mutational burden as biomarkers. $J$ Clin Oncol. 2019;37(12):922-1000.

20. Sun JX, He Y, Sanford E, et al. A computational approach to distinguish somatic vs. germline origin of genomic alterations from deep sequencing of cancer specimens without a matched normal. PLoS Comput Biol. 2018;14(2):e1005965.

21. Buchhalter I, Rempel E, Endris V, et al. Size matters: dissecting key parameters for panel-based tumor mutational burden (TMB) analysis. Int $J$ Cancer. 2019;144(4):848-858. 
22. Ascierto PA, Bifulco C, Palmieri G, Peters S, Sidiropoulos N. Preanalytic Variables and Tissue Stewardship for Reliable Next-Generation Sequencing (NGS) Clinical Analysis. J Mol Diagn. 2019;21(5):756-767.

23. Buttner R, Longshore JW, Lopez-Rios F, et al. Implementing TMB measurement in clinical practice: considerations on assay requirements. ESMO Open. 2019;4(1):e000442.

24. Kazdal D, Endris V, Allgauer M, et al. Spatial and temporal heterogeneity of panelbased tumor mutational burden (TMB) in pulmonary adenocarcinoma: separating biology from technical artifacts. J Thorac Oncol. 2019;14(11):1935-1947.

25. Stenzinger A, Allen JD, Maas J, et al. Tumor mutational burden (TMB) standardization initiatives: recommendations for consistent TMB assessment in clinical samples to guide immunotherapy treatment decisions. Genes Chromosomes Cancer. 2019;58(8):578-588.

26. Kerr KM, Tsao M-S, Nicholson AG, Yatabe Y, Wistuba II, Hirsch FR. Programmed death-ligand 1 immunohistochemistry in lung cancer: in what state is this art? $J$ Thorac Oncol. 2015;10(7):985-989.

27. Chang H, Sasson A, Srinivasan S, et al. Bioinformatic methods and bridging of assay results for reliable tumor mutational burden assessment in non-small cell lung cancer. Mol Diagn Ther. 2019;23(4):507-520. 
28. Alborelli I, Leonards K, Rothschild SI, et al. Tumor mutational burden assessed by targeted NGS predicts clinical benefit from immune checkpoint inhibitors in nonsmall cell lung cancer. J Pathol. 2020;250(1):19-29 .

29. Do H, Molania R, Mitchell PL, Vaiskunaite R, Murdoch JD, Dobrovic A. Reducing Artifactual EGFR T790M Mutations in DNA from Formalin-Fixed Paraffin-Embedded Tissue by Use of Thymine-DNA Glycosylase. Clin Chem. 2017;63(9):1506-1514.

30. Heeke S, Benzaquen J, Long-Mira E, et al. In-house Implementation of Tumor Mutational Burden Testing to Predict Durable Clinical Benefit in Non-small Cell Lung Cancer and Melanoma Patients. Cancers (Basel). 2019;11(9). 


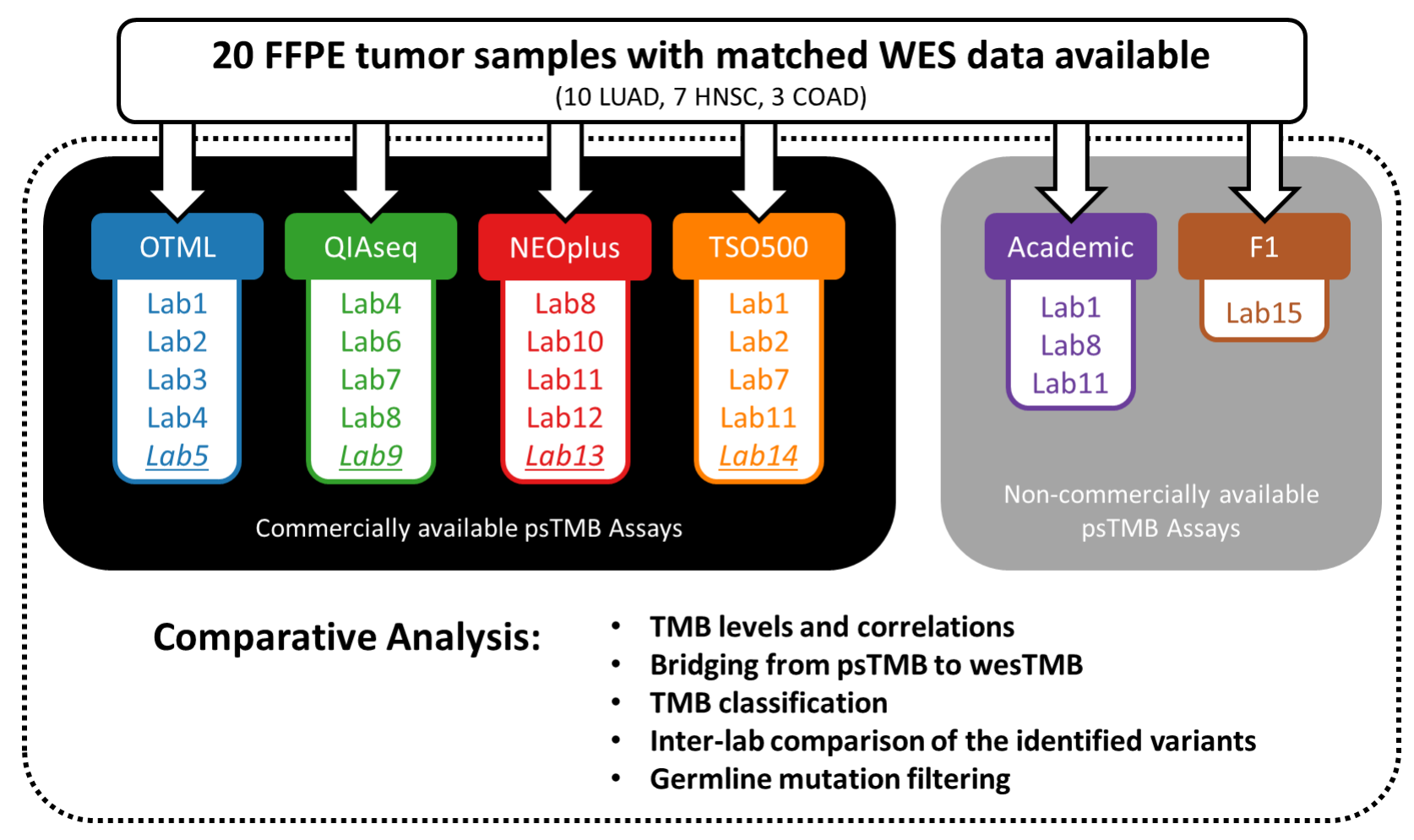

Figure 1 
$A$

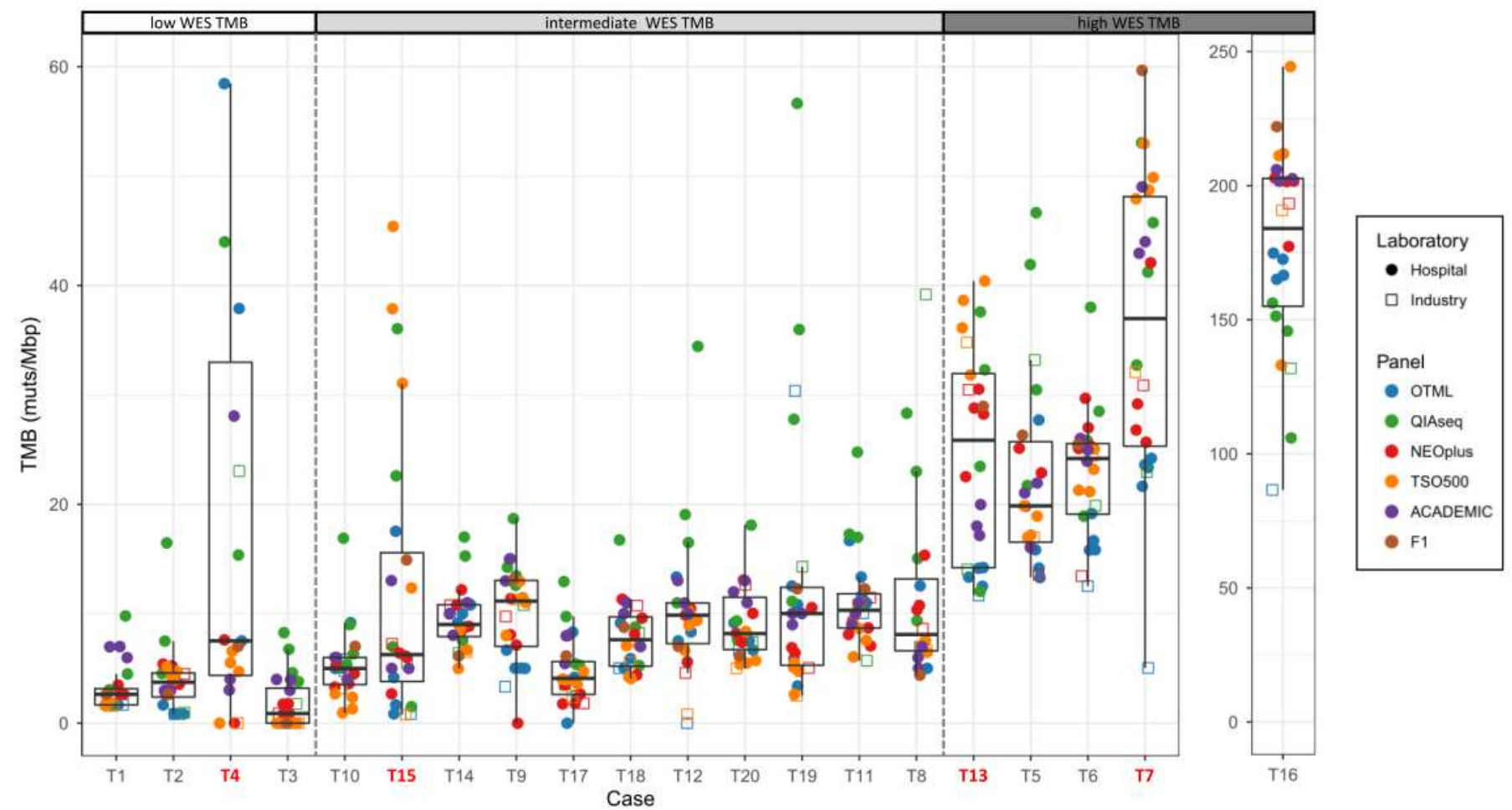

B

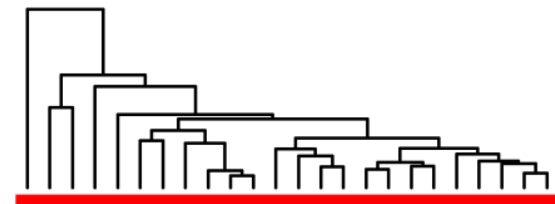

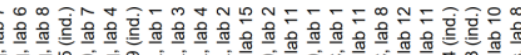

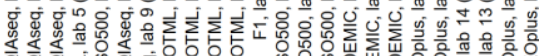

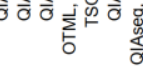

C

TMB (muts/Mbp)

- $>40$

- $20-40$

- $10-20$

- $2.5-5$

- $1.25-2.5$

- $<1.25$
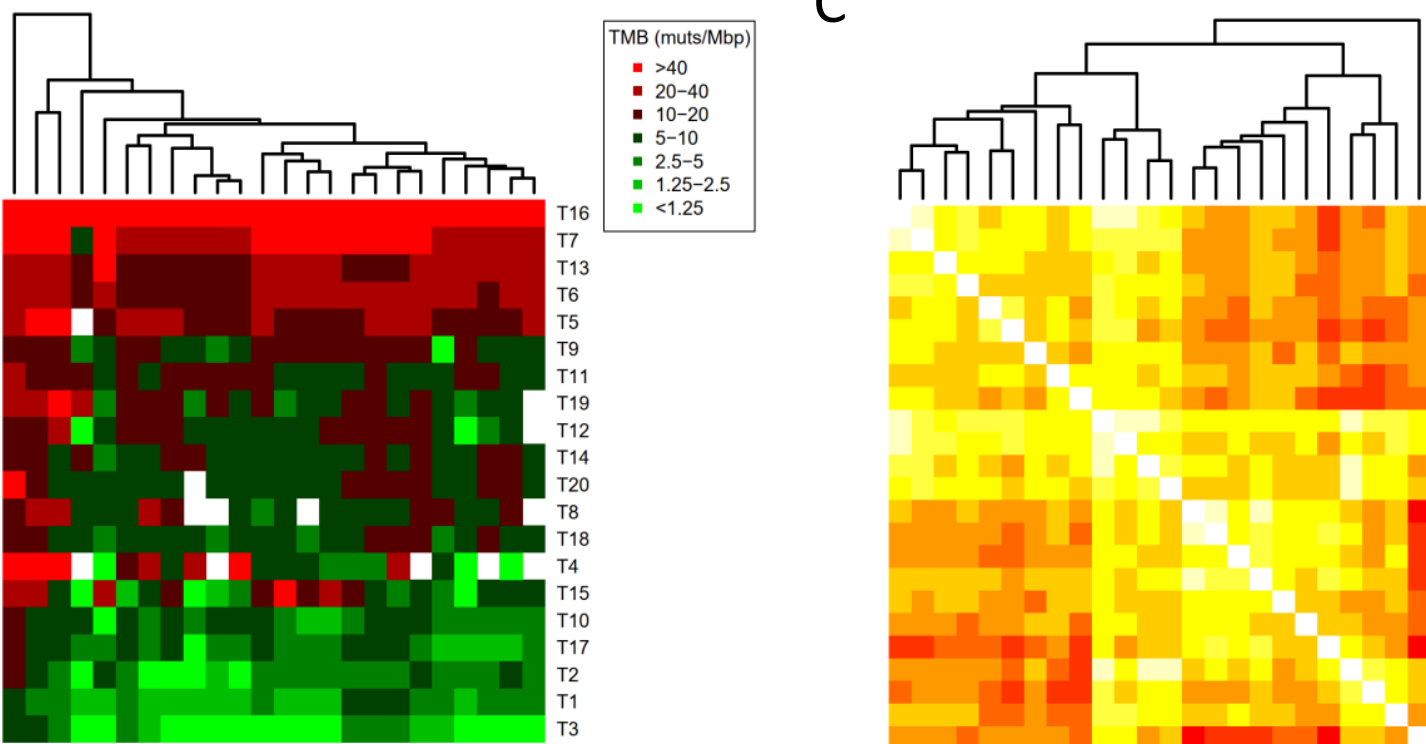

OTML, lab 2

OTML, lab 3

QIAseq, lab 4

ACADEMIC, lab 8

QIAseq, lab 9 (ind.)

QIAseq, lab 8

NEOplus, lab 11

QIAseq, lab 6

QIAseq, lab 7

NEOplus, lab 8

OTML, lab 4

NEOplus, lab 13 (ind.)

NEOplus, lab 12

SO500, lab 2

SO500, lab 1

ACADEMIC, lab

TSO500, lab 11

F1, lab 15

OTML, lab 1

TSO500, lab 7

NEOplus, lab 10

TSO500, lab 14 (ind.)

ACADEMIC, lab 11

OTML, lab 5 (ind.)

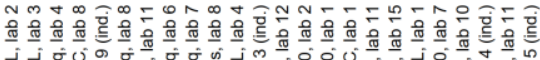

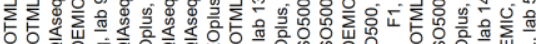

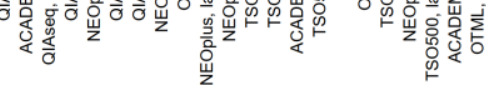

Figure 2 
A
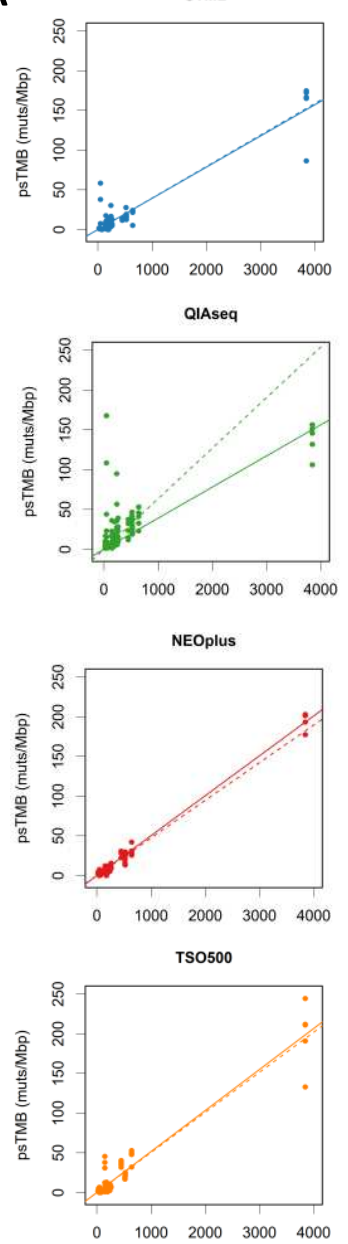

ACADEMIC

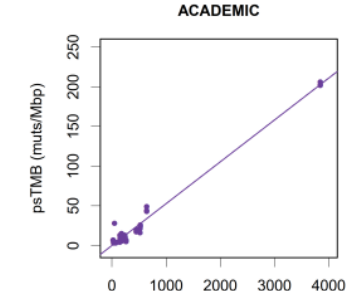

F1

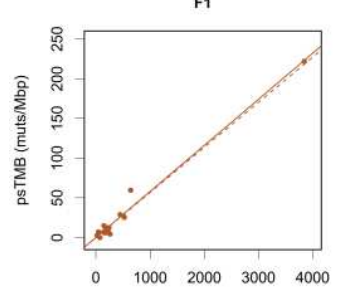

wesTMB (muts)
B
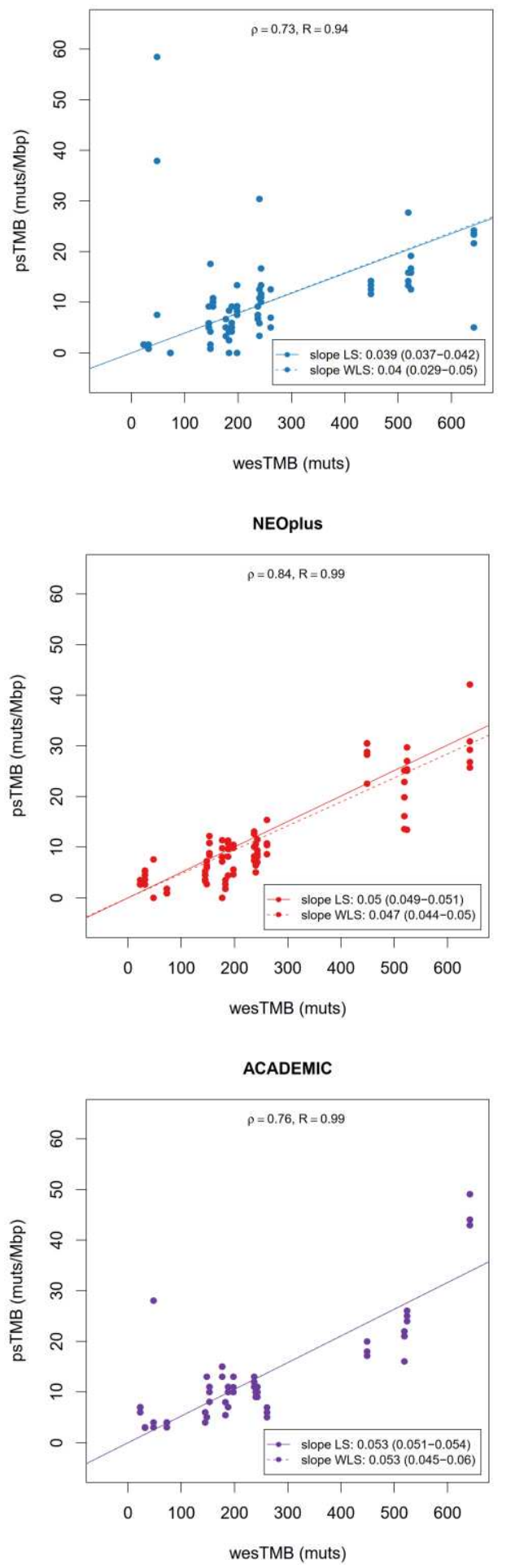

QIAseq

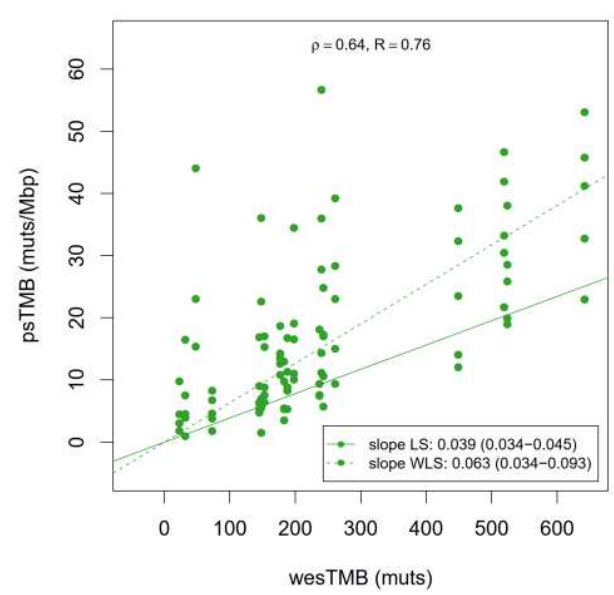

TSO500

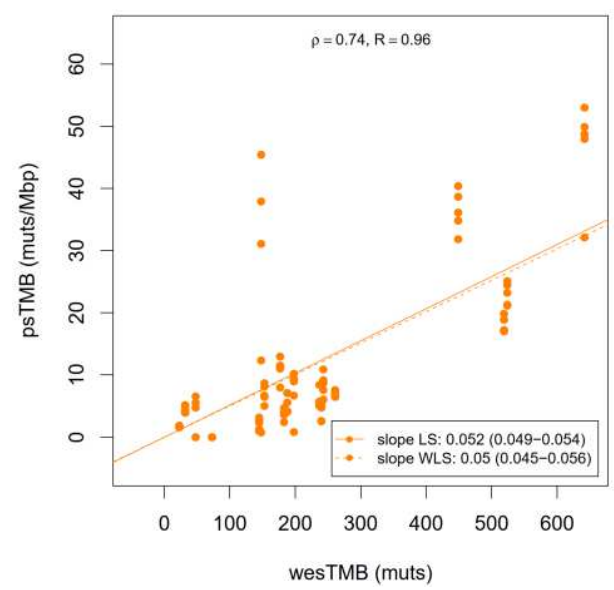

F1

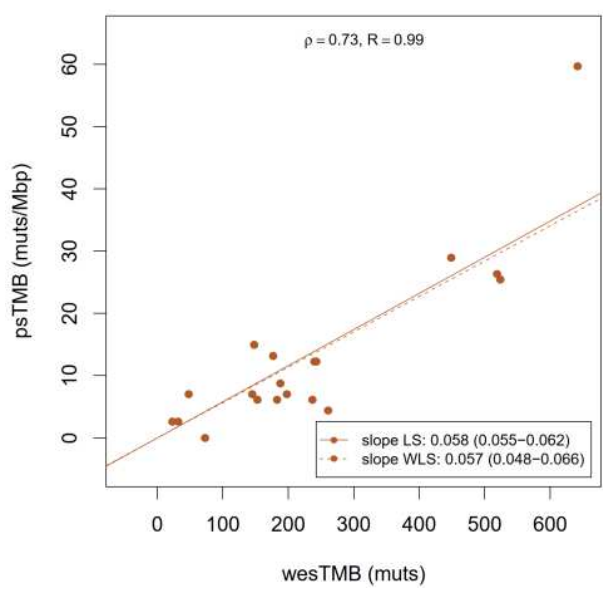

Figure 3 
A

2-tier classification, cutpoint: 199 muts

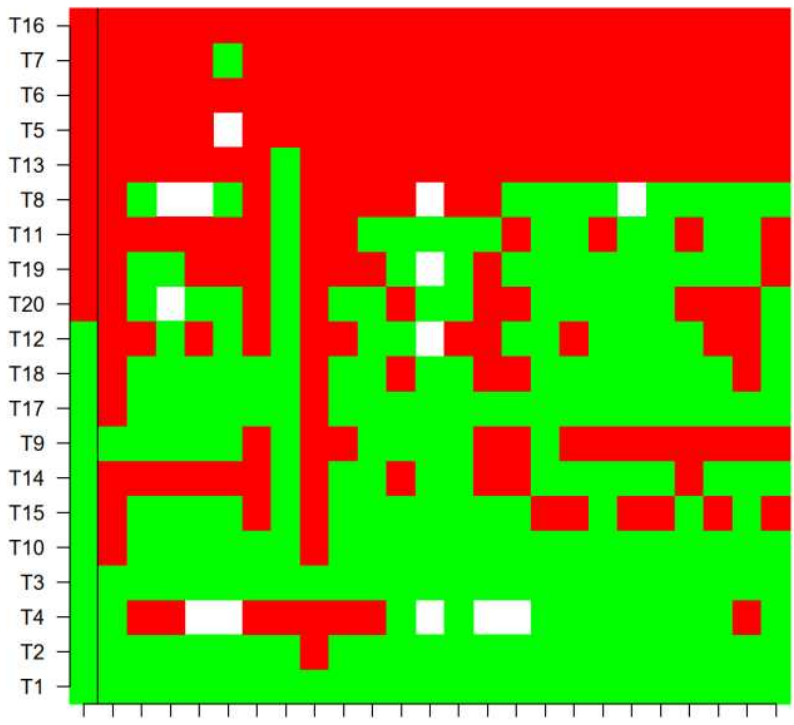

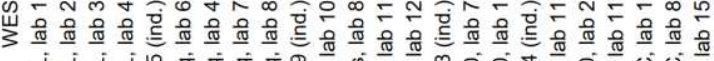

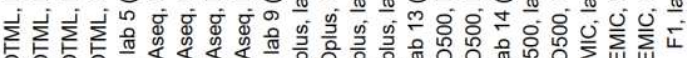

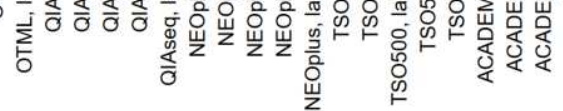

B

3-tier classification, cutpoints: 100 muts and 300 muts

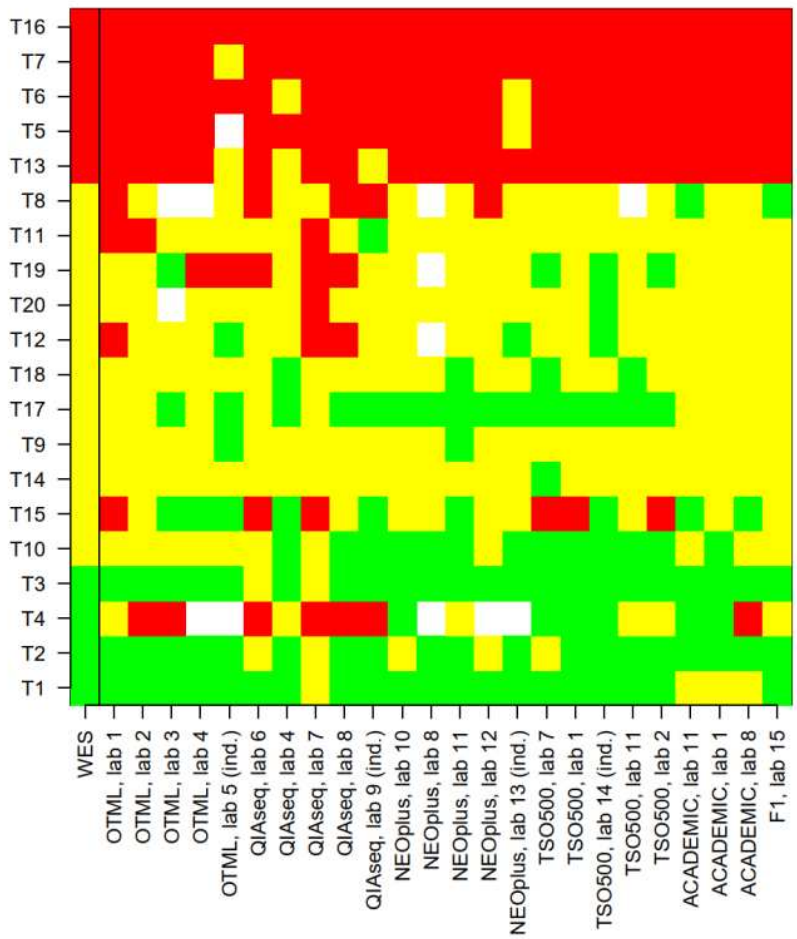

Figure 4 
A

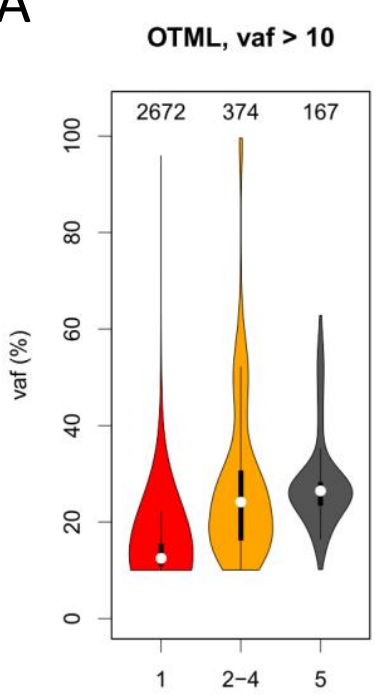

number of labs
QIAseq, vaf > 5

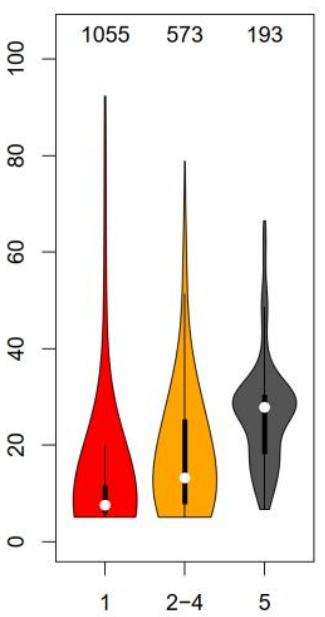

number of labs
NEOplus, vaf $>10$

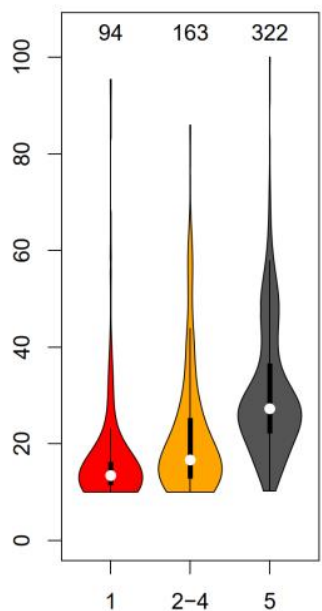

number of labs
TSO500, vaf > 5

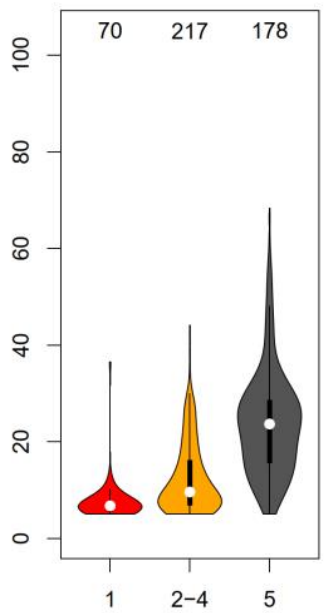

number of labs
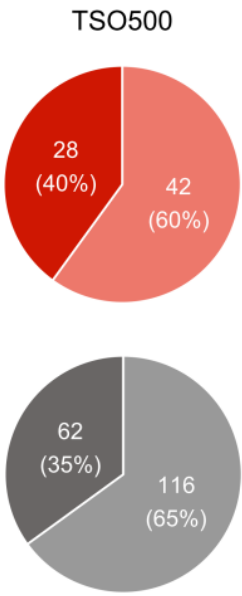

ACADEMIC, vaf $>5$

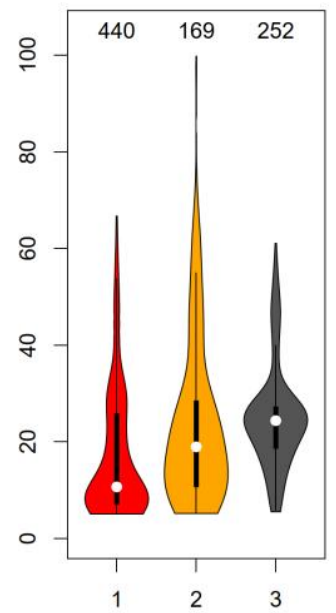

number of labs

ACADEMIC

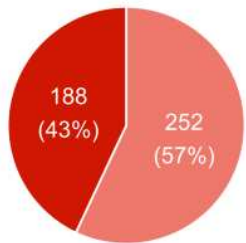

114

$(45 \%)$

138

$(55 \%)$

$C>T / G>A$

107
$(64 \%)$

no $C>T / G>A$
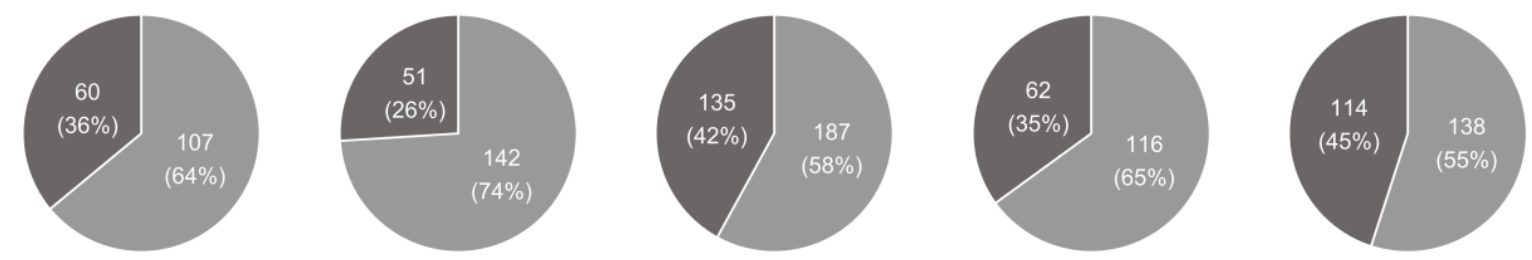

C

C Median Coverage

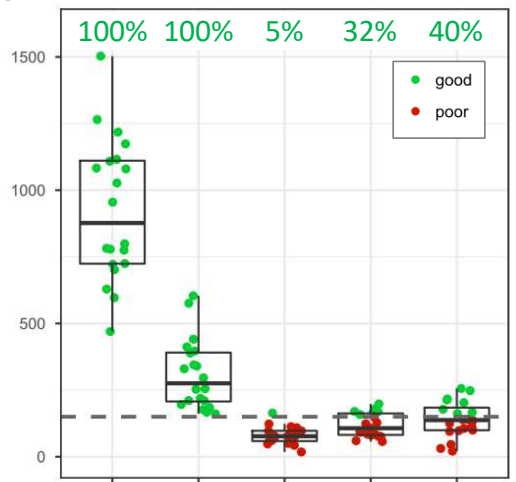

Lab 1 Lab 2 Lab 7 Lab 11 Illumina
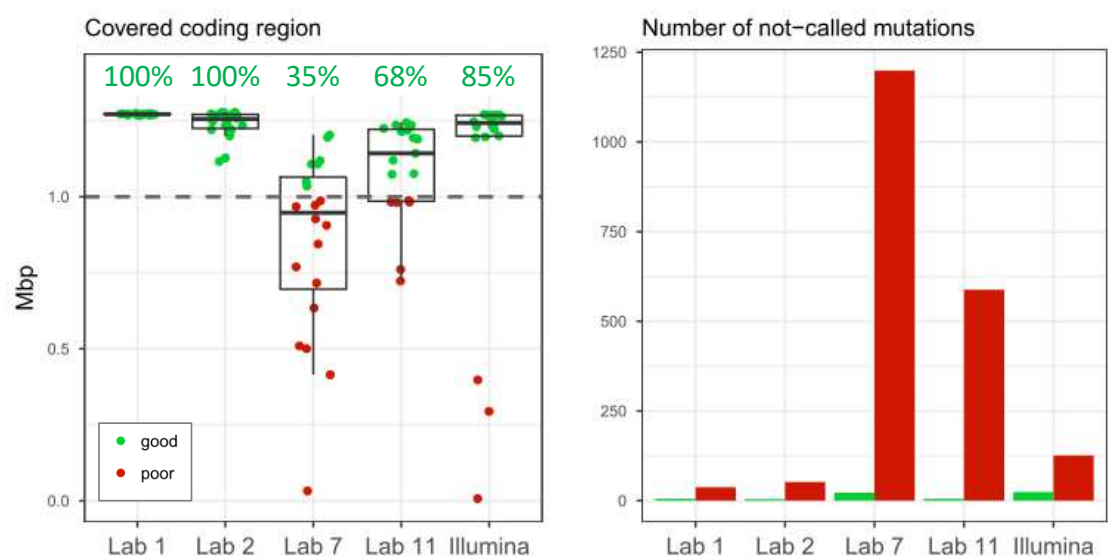

Coverage

$>200 x$
$<200 x$

Figure 5 

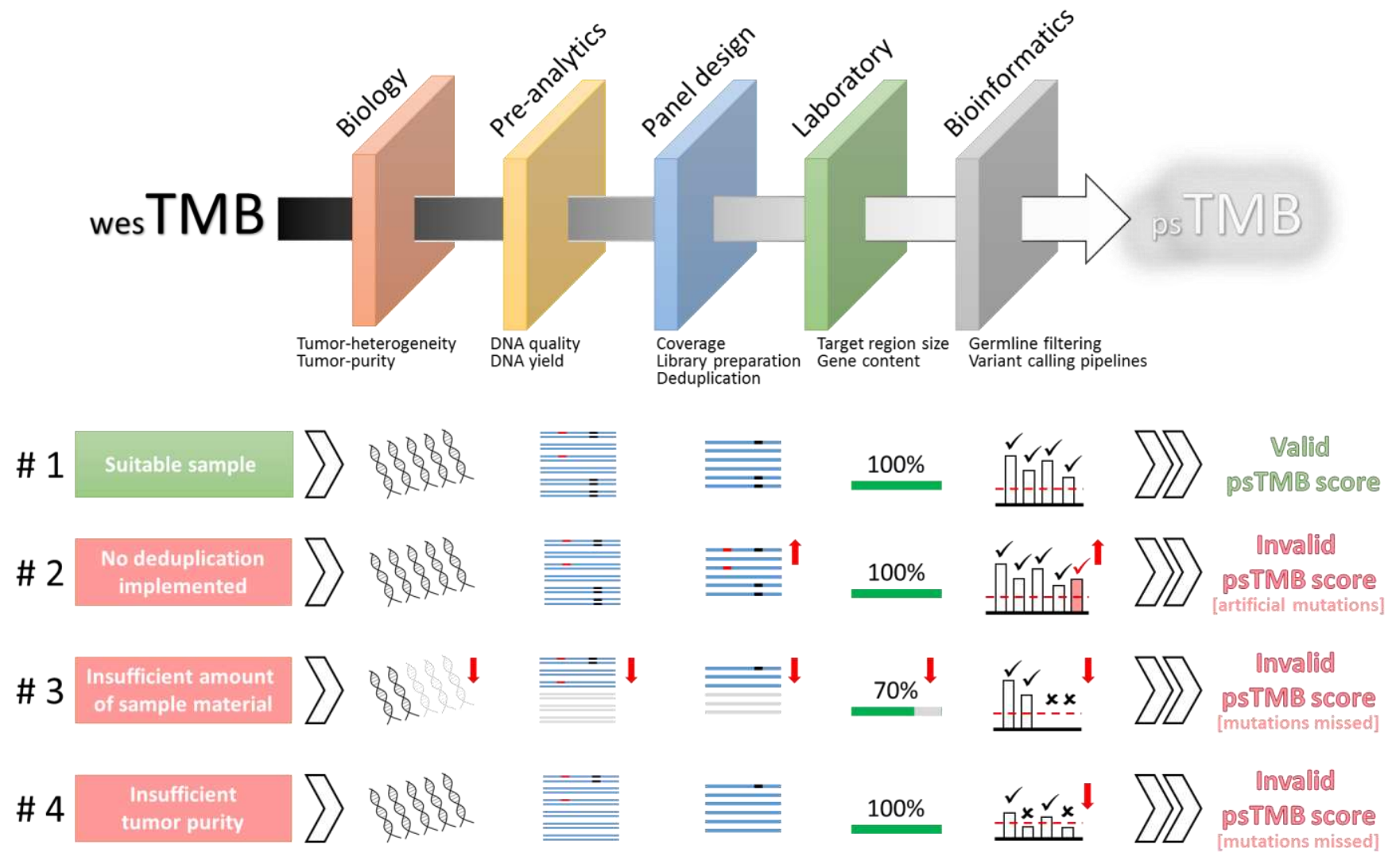

Figure 6 


\section{Supplementary Material}

Suppl. 1. Origin, TCC, and DNA quality of the analyzed tumor tissue samples. COAD, colon adenocarcinoma; HNSCC, head and neck squamous cell carcinoma; LUAD, lung adenocarcinoma; MSI-H, high-level microsatellite instability; TCC, tumor cell content.

Suppl. 2. Overview of the sequencing panels evaluated for tumor mutational burden measurement and detailed methodological information for panel and whole exome sequencing approaches.

Suppl. 3. Distribution of tumor mutational burden assessed by whole exome sequencing in LUAD, LUSC, HNSCC, and COAD. Data from The Cancer Genome Atlas. COAD, colon adenocarcinoma; HNSCC, head and neck squamous cell carcinoma; LUAD, lung adenocarcinoma; LUSC, lung squamous cell carcinoma.

Suppl. 4. Intra-laboratory variability for each of the tested sequencing panels. Mean psTMB with error bar equal to the psTMB SD. Top line: CV. ACADEMIC, customdesigned academic panel; CV, coefficient of variation; muts/Mbp, mutations per megabase pair; NEOplus, NEOplus RUO assay; OTML, Oncomine Tumor Mutational Load Assay; psTMB, TMB assessed by gene panel sequencing; QIAseq, QIAseq TMB panel; SD, standard deviation; TMB, tumor mutational burden; TSO500, TruSight Oncology 500 panel. 
Suppl. 5. Inter-laboratory and inter-assay correlations (Pearson R) between levels of TMB assessed by gene panel sequencing and TMB assessed by whole exome sequencing. (A) Study cohort $(n=20)$. (B) Study cohort without sample T16 (sample with very high TMB, $\mathrm{n}=19$ ). ACADEMIC, custom-designed Academic panel; $\mathrm{F} 1$, FoundationOne assay; NEOplus, NEOplus RUO assay; OTML, Oncomine Tumor Mutational Load Assay; QIAseq, QIAseq TMB panel; TMB, tumor mutational burden; TSO500, TruSight Oncology 500 panel.

Suppl. 6. Conversion of wesTMB values to psTMB values and vice versa. BF: factor to multiply psTMB for conversion to wesTMB. LS: BF from LS. WLS: BF from WLS. ACADEMIC, custom-designed academic panel; BF, bridging factor; F1, FoundationOne assay; LS, least squares; NEOplus, NEOplus RUO assay; OTML, Oncomine Tumor Mutational Load Assay; psTMB, TMB assessed by gene panel sequencing; QIAseq, QIAseq TMB panel; TMB, tumor mutational burden; TSO500, TruSight Oncology 500 panel; wesTMB, TMB assessed by whole exome sequencing; WLS, weighted least squares.

Suppl. 7. Three-tier classification using the cutpoints of 150 and 250 mutations. Red: high TMB; yellow: intermediate TMB; green: low TMB. Strong misclassifications (i.e., misclassifications mixing TMB high and TMB low cases): 5.8\%. Weak misclassifications (i.e., misclassifications mixing intermediate TMB cases with TMB high or TMB low cases): 25.9\%. ACADEMIC, custom-designed academic panel; F1, FoundationOne assay; NEOplus, NEOplus RUO assay; OTML, Oncomine Tumor Mutational Load 
Assay; QIAseq, QIAseq TMB panel; TMB, tumor mutational burden; TSO500, TruSight Oncology 500 panel; WES, whole exome sequencing.

Suppl. 8. Performance of germline filtering for the mutations detected by a panel of 1.34 Mbp in ten LUAD samples. In silico database filtering is compared with filtering by sequencing of paired normal (germline) samples. (A) Sensitivity, specificity, accuracy, PPV, and NPV of the classification as somatic mutation in 1516 mutations (pooled analysis of ten samples). (B-E) TMB error resulting from false somatic and false germline classifications for each of the 10 tumors. MAF, minor allele frequency; muts/Mbp, mutations per megabase pair; NPV, negative predictive value; PPV, positive predictive value; TMB, tumor mutational burden.

Suppl. 9. As Suppl. 8, but for the germline mutation filtering pipeline developed by Illumina for the TSO500 panel. (A) Comparison of the Illumina pipeline with filtering by gnomAD, ExAC, and dbSNP. (B) Illumina pipeline, database filtering only. (C) Illumina pipeline, database and proxy filtering. MAF, minor allele frequency; muts/Mbp, mutations per megabase pair; NPV, negative predictive value; PPV, positive predictive value; TMB, tumor mutational burden. 